\title{
Secondary Electrons in EUV Lithography
}

\section{Justin Torok, ${ }^{\text {a }}$ Ryan Del Re, ${ }^{\text {a Henry Herbol, }}{ }^{\text {a }}$ Sanjana Das, ${ }^{\text {a }}$ Irina Bocharova, ${ }^{\text {a }}$ Angela Paolucci, ${ }^{\mathrm{a}}$ Leonidas E. Ocola, ${ }^{\mathrm{b}}$ Carl Ventrice Jr., ${ }^{\mathrm{a}}$ Eric Lifshin, ${ }^{\mathrm{a}}$ Greg Denbeaux ${ }^{\mathrm{a}}$ and Robert L. Brainard ${ }^{\mathrm{a}}$}

\author{
a. College of Nanoscale Science and Engineering, University at Albany \\ b. Argonne National Laboratories
}

\begin{abstract}
Secondary electrons play critical roles in several imaging technologies, including extreme ultraviolet (EUV) lithography. At longer wavelengths of light (e.g. 193 and $248 \mathrm{~nm}$ ), the photons are directly involved in the photochemistry occurring during photolysis. EUV light $(13.5 \mathrm{~nm}, 92 \mathrm{eV})$, however, first creates a photoelectron, and this electron, or its subsequent daughter electrons create most of the chemical changes that occur during exposure. Despite the importance of these electrons, the details surrounding the chemical events leading to acid production remain poorly understood. Previously reported experimental results using high PAG-loaded resists have demonstrated that up to five or six photoacids can be generated per incident photon. Until recently, only electron recombination events were thought to play a role in acid generation, requiring that at least as many secondary electrons are produced to yield a given number of acid molecules. However, the initial results we have obtained using a Monte Carlo-based modeling program, LESiS, demonstrate that only two to three secondary electrons are made per absorbed EUV photon. A more comprehensive understanding of EUVinduced acid generation is therefore needed for the development of higher performance resists.

Keyword: secondary electron, EUV lithography, photoelectron, high PAG-loaded resist
\end{abstract}

\section{Introduction}

As the critical feature size for integrated circuit manufacturing continues to shrink, the wavelength of light needed to pattern these features is also decreasing. With 193-nm lithography reaching its resolution limit, the leading candidate to replace it for future nodes is extreme ultraviolet (EUV, $\gg=13.5 \mathrm{~nm}$ ) lithography. However, there are several significant challenges for EUV, not the least of which is the design of new photoresists capable of continued improvement in resolution, lineedge-roughness (LER), and sensitivity. One of the key differences between traditional photolithography and EUV is the fundamental mechanism by which photons used in these imaging technologies interact with matter. At conventional wavelengths $(365,248$, and 193 $\mathrm{nm})$, a photon promotes an electron in a chromophore within the resist from a bonding to an anti-bonding orbital, which causes these molecules to decompose and produce acid $[1,2]$.
In contrast, EUV photons have enough energy to ionize resist molecules, and it is the resulting photoelectrons that initiate the chain of events leading to acid production. Therefore, it is essential to understand the mechanistic details of the interactions of low-energy electrons with resists in order to optimize the performance of EUV lithography.

One description of the process by which photons create acids is shown in Figure 1. As an EUV photon $(92 \mathrm{eV})$ is absorbed, it creates a photoelectron with a kinetic energy of approximately 78-86 eV [3-5]. As it scatters throughout the polymer matrix, this electron loses energy through several different mechanisms (see Section 3.1 below), one of which is the initiation of further ionization events. In this way, each EUV photon has the potential to generate several lower-energy secondary electrons. However, the details surrounding the chemical events leading to acid generation are still a matter of speculation, and 
while our group [6] and the groups of Kozawa and Tagawa [7-10] have attempted to examine this mechanism with various theoretical and experimental approaches, the details of these chemical events remain poorly understood.

Dozens of studies have been published on the interactions of high-energy photons (e.g. xrays $[11,12])$ with matter over several decades. Additionally, there have been many excellent studies of electron-solid interactions for a broad range of energies (20 eV-100 keV) [13-22]. This body of work has provided a foundation upon which our work with EUV has been built; however, the work we and Kozawa have done is quite distinct from previous studies in that the energy of EUV photons, and the secondary electrons created by these photons, is in a much lower energy range than most of the fundamental theoretical and experimental work. We are interested in electron-resist interactions for electrons with energies ranging from 5 to 90 $\mathrm{eV}$, where to our knowledge very little work has been done. In particular, it is recognized that conventional energy loss equations used in Monte Carlo calculations for high-energy electrons, such as the Bethe equation, do not apply to electrons in this energy range [13]. The focus of our work is to evaluate and understand the roles played by secondary electrons in EUV lithography.

In this work, we have modified a published Monte Carlo-based simulation program that was originally developed for high energy photons (800-1000 eV) and high energy electrons (30$100 \mathrm{keV}$ ) so that it will simulate the relatively low energy events occurring in EUV resists. This program, called LESiS (Low-energy Electron Scattering in Solids) [23,24], was specifically designed to track the trajectories of individual electrons based on their probabilities of interacting with nearby atoms within matter, as described below in Section 3. The updated version of this program, v7.71, tracks electrons with kinetic energies down to $1 \mathrm{eV}$ as they interact both inelastically and elastically with the surrounding resist matrix. Using this approach, we are able to determine the locations and energies of the secondary electrons generated and therefore approximate the total electron yield per EUV photon as well as the extent of electron penetration in the resist film.

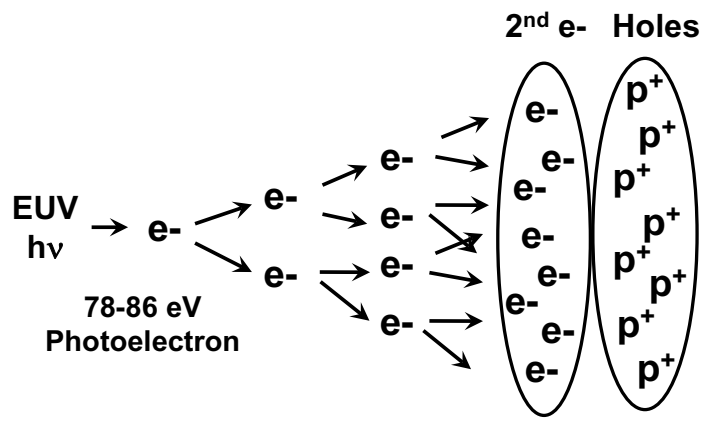

Figure 1. Qualitative scheme depicting multiple electrons being created after the absorption of an EUV photon.

\section{Film Quantum Yields and Mechanistic Analysis}

In this section, we discuss three potential mechanisms for photoacid generation and previous results from two PAG-loading studies that explore the efficiency of EUV photons in generating acid, which we define as the film quantum yield (FQY): the number of acids produced per incident photon. These three mechanisms have been described previously in the lithographic [3,6-8,25-27] or physics $[13,28,29]$ literature. However, recognizing that different disciplines describe similar topics in vastly different ways, we are revisiting all of these topics in this paper so that a balanced discussion can be presented in the language of chemistry.

\subsection{Two Studies of Film Quantum Yield.}

Two complementary studies provide important information about the role of secondary electrons in converting PAGs to acid (Figure 2) $[4,6,8,30]$. In both studies, the film quantum yields of acid generation were determined as a function of PAG loading. Although the exact structures of the PAGs were slightly different, the key findings in each study were in agreement: (1) iodonium PAGs give the 
best film quantum yields (max FQY $(\Phi)$ : 5.6 and 4.9 acids/absorbed photon); (2) followed by sulfonium PAGs ( $\max \Phi=4.3$ and 3.3 ); (3) followed by non-ionic PAGs ( $\max \Phi=2.9$ and 3.1) for Higgins et al. [6] and Kozawa et al. [30], respectively.

\subsection{Mechanism 1}

One mechanism for EUV-induced acid conversion involves the generation of secondary electrons as shown in Figures 1 and 3A. According to this mechanism, several secondary electrons are created from the initial photoelectron, and these daughter electrons react with photoacid generators to produce acid (Figure 3A). Recent results by Thackeray et al. show that the EUV sensitivity is proportional to the reduction potential of PAGs, which is consistent with Mechanism 1 [31]. This mechanism is also consistent with the experimental results shown in section 2.1 in which increased PAG loading produces an increase in FQY (Figure 2) [3,4,6,8,30].

Furthermore, Higgins and coworkers designed a simple kinetic model of the interaction of the electrons and holes in which the electrons either react with PAGs $\left(\mathrm{k}_{1}\right)$ or recombine with holes $\left(\mathrm{p}^{+}, \mathrm{k}_{2}\right.$, Figure $\left.3 \mathrm{~A}\right)$ [6]. Fitting the results from these three PAGs produced ratios of rate constants $\left(\mathrm{k}_{2} / \mathrm{k}_{1}\right)$ of 136 , 195, and 230 for the reaction with iodonium, sulfonium and non-ionic PAGs, respectively. Therefore, based on this analysis, the rate constant for electron-hole recombination is more than two orders of magnitude larger than electron-induced acid generation.

\subsection{Mechanism 2 - Kozawa and Tagawa et al.}

Kozawa, Tagawa and coworkers have published extensively on subjects related to the interactions of photons and electrons with matter. The earliest work in this area predates that of Higgins and coworkers by approximately twenty years and is concerned with the interaction of compounds in solution with $28 \mathrm{MeV}$ gamma
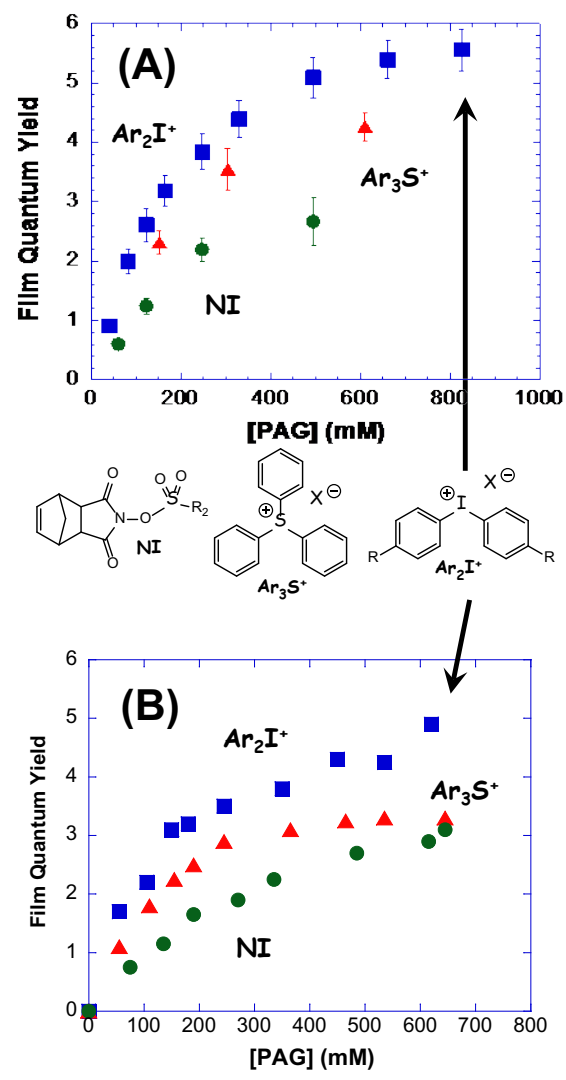

Figure 2. Film quantum yield studies. Two studies determined the FQY as a function of PAG concentration. (A) Higgins and Brainard et al. reached a maximum of 5.6 acids/photon $(\mathrm{R}=$ $\left.\mathrm{C}\left(\mathrm{CH}_{3}\right)_{3}, \mathrm{X}=\mathrm{C}_{4} \mathrm{~F}_{9} \mathrm{SO}_{3}{ }^{-}, \mathrm{R}_{2}=\mathrm{C}_{4} \mathrm{~F}_{9}\right)$ [6]. (B) Kozawa and Tagawa et al. reached a maximum of 4.9 acids/photon $\left(\mathrm{R}=\mathrm{H}, \mathrm{X}=\mathrm{CF}_{3} \mathrm{SO}_{3}{ }^{-}, \mathrm{R}_{2}=\mathrm{CF}_{3}\right)$ [30].

rays [7]. Our intent is not to review this extensive body of work, but to compare and contrast a couple of its key features with the other mechanisms discussed in this paper.

An important factor in Kozawa's and Tagawa's work is the role of phenolic radical cations in the generation of acid (Mechanism 2, Figure 2B). They propose that the major pathway of acid generation is through the ionization of phenolic moieties in the polymer, creating a radical cation and a free electron. Disproportionation between this radical cation and another phenol produces a neutrall radical 
(A) Mechanism 1

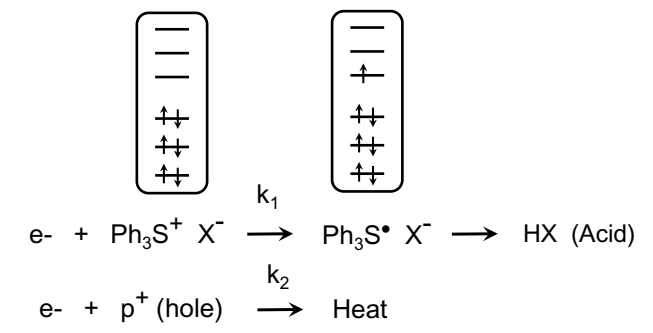

(B) Mechanism 2

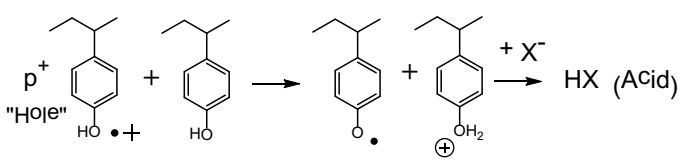

Figure 3. Two proposed mechanisms. (A) Mechanism 1, in which an electron adds to an empty orbital of a photoacid generator and causes it to decompose, and (B) Mechanism 2, in which the creation of a secondary electron creates a radical cation (or hole, $\mathrm{p}^{+}$) which reacts with a second phenol to create acid.

and a protonated phenol; this species is strongly acidic and ultimately donates a proton to a free PAG counter anion to form the catalytic strong acid (Figure 3B) [7-9]. The PAG, then, is an electron trap, decreasing the frequency of electron recombination with nearby phenolic radical cations and thereby increasing the probability of the above mentioned disproportionation reaction [7,8]. Additionally, these researchers indicate that while direct interaction between secondary electrons and PAGs can generate acid (Mechanism 1, Figure $3 \mathrm{~A})$, they estimate that this mechanism only accounts for about $10 \%$ of the photo-generated acid $[8,32]$.

2.4 Comparison of Mechanisms 1 and 2 with Yields of Acids.

Assuming the secondary electron cascade to be correct (Figure 1), the original impetus for the Higgins study was to set a lower limit to the number of electrons that are made. The conclusion based on the maximum quantum yield value of 5.6 was, therefore, that at least 5-6 electrons are created and react with PAG to form acid at the very minimum. Yet, realizing that yields in chemistry are rarely $100 \%$ and that other reactions may occur which will annihilate secondary electrons without creating acid (e.g. electron-hole recombination), the number of secondary electrons generated per photon would be greater than five and perhaps as many as nine or ten if Mechanism 1 is the dominant acidproduction pathway.

Mechanism 2 starts out the same way as Mechanism 1, in that an initial photoelectron generates a cascade of secondary electrons and their holes (Figure 1). Mechanism 2, however, diverges from Mechanism 1 in that the principal process for creating acid involves the phenolic radical cation (hole or $\mathrm{p}^{+}$, Figure $3 \mathrm{~B}$ ). Based on this mechanism, the number of holes generated (and therefore the number of secondary electrons) would also have to exceed the maximum number of acids generated in the ultrahigh-PAG experiments when other energy loss mechanisms are taken into account. Therefore, the number of electron/hole pairs necessary is the same as for Mechanism 1.

\subsection{Mechanism 3 - Internal Excitation}

Here we would like to propose a third mechanism that may be responsible for the decomposition of PAGs to acids. This mechanism is based on an electronic transition that is known in the physics and spectroscopic literature variously as "internal excitation", "electronic excitation", or "molecular exciton formation". For clarity, we will only use "internal excitation" in this paper. This is an energy loss mechanism by which a passing electron excites an electron in a bonding orbital of a molecule or polymer functional group to an internal anti-bonding orbital. As a result, the free secondary electron loses energy equal to the energy gap between states - this can be as low as 2-5 eV [25,26,33-35]. Qualitatively speaking, the electronic transition that the PAG undergoes is very similar to the transition that occurs when a PAG absorbs a photon during ultraviolet spectroscopy or photolysis. As with photonbased exposure mechanisms, this 
HOMO/LUMO transition should cause the excited state of the PAG to undergo homolytic cleavage, followed by several additional steps to produce acid (Figure 4). Although this internal excitation mechanism has been described in the physics literature for a variety of molecules and materials, including PMMA [25,26,33], the concept of PAGs decomposing by this mechanism in chemically-amplified EUV photoresists is relatively new [5,27].

One aspect of this mechanism that is particularly appealing is that it uses energy coming from the EUV photon more efficiently than do Mechanisms 1 and 2. They require multiple ionization steps to create multiple electron/hole pairs, with each ionization step consuming about 8.8-10 eV energy. On the other hand, the bonds that typically break during photolysis of a PAG are about $60 \mathrm{kcal} / \mathrm{mol}$, or 2.5-3 eV. Therefore, based on this mechanism, a single EUV photoelectron of $\sim 80 \mathrm{eV}$ should have sufficient energy to induce as many as 20$30 \mathrm{HOMO} / \mathrm{LUMO}$ transitions in the resist, each of which could initiate a chain of chemical events leading to acid production.

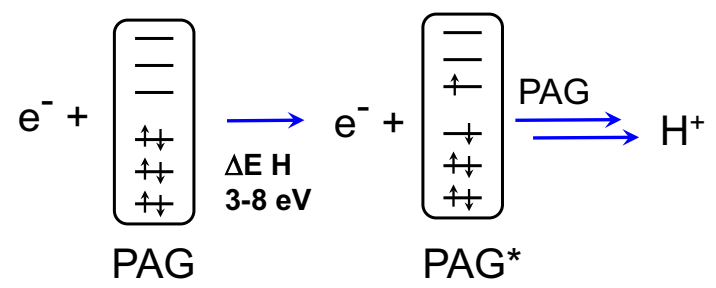

Figure 4. A qualitative description of Mechanism 3. A passing electron loses 3-8 eV of energy to excite an electron in a bonding orbital to an anti-bonding orbital. This transition is known in the spectroscopy literature as internal excitation. Other than the fact that the electron is not destroyed, but just loses some of its energy, this process is very similar to the absorption of a photon by a chromophore.

\section{Modeling with LESiS}

One key piece of our strategy for understanding the role of secondary electrons in EUV is to complement experimental results (e.g. from e-beam depth penetration studies, EELS and photo-electron spectroscopy) with those of a specialized Monte Carlo modeling program that simulates exposures. We have started with the LESiS (Low-energy Electron Scattering in Solids) modeling program that was originally developed to study secondary electrons resulting from interaction of high-energy photons (10$8000 \mathrm{eV})$ and high-energy electrons (30-100 $\mathrm{keV})$ with matter $[23,24]$. For our work with EUV, the code was extended to track traveling electrons down to $1 \mathrm{eV}$ (from $30 \mathrm{eV}$ ), and secondary electron yield for aluminum was used as a benchmark.

\subsection{Energy Loss Mechanisms.}

The interaction of the incident EUV photons with photoresists results in the ionization of valence electrons within the photoresist. The binding energies of these valence electrons depend upon chemical structures of the resist's constituent molecules. For most hydrocarbons, binding energies are about 6-14 eV. Therefore, the EUV photons will generate photoelectrons with kinetic energies in the range of 78-86 eV [4]. Electrons within this energy range can interact with molecules in the photoresist by one of five main processes: elastic scattering, ionization, plasmon generation, internal excitation, or vibrational excitation (Figure 5) [28,29,33-35]. (1) Elastic scattering occurs when a free electron is deflected by the electrostatic attraction of the positively-charged atomic core without energy transfer. The scattering angle, or momentum transfer, is usually small (around $10^{\circ}$ ) for this type of interaction, but can be as great as $180^{\circ}$. (2) Ionization occurs when the free electron causes a bound electron to leave the molecule thereby creating a secondary electron and a hole. The original electron loses energy equivalent to the binding energy (6-14 eV) plus the kinetic energy of the liberated secondary electron, which can be 
large enough for this daughter electron to cause further ionization events. Conversely, in the process of photoionization, the incident photon transfers all of its energy to the photoelectron and then ceases to exist. (3) A plasmon is a collective oscillation of the valence electrons as typically defined for metals. In polymers a model described by the dielectric function is needed to understand the presence of a plasmon. The dielectric function is expressed as a sum of oscillators. Some polymers exhibit an oscillator with energies around 5-7 eV, which corresponds to a $\pi$ plasmon. Most polymers exhibit a set of oscillators in the 20-30 eV energy range corresponding to the $\sigma+\pi$ plasmon [36]. These plasmons correspond to carbon atoms in the $\sigma$ and $\pi$-states [37]. The energy range for the $\sigma+\pi$ plasmon is typical of what can be described using a Jellium model. (4) The electron can lose energy during a process called internal excitation described in section 2.5 above. This is essentially the same process shown in Figure 4 for PAGs but can occur with any of the molecules or polymer side groups within the resist film. (5) Lastly, for electrons with energies below the threshold for internal excitations, the primary loss mechanism is through vibrational excitation, in which an electron promotes the transition of a molecule to

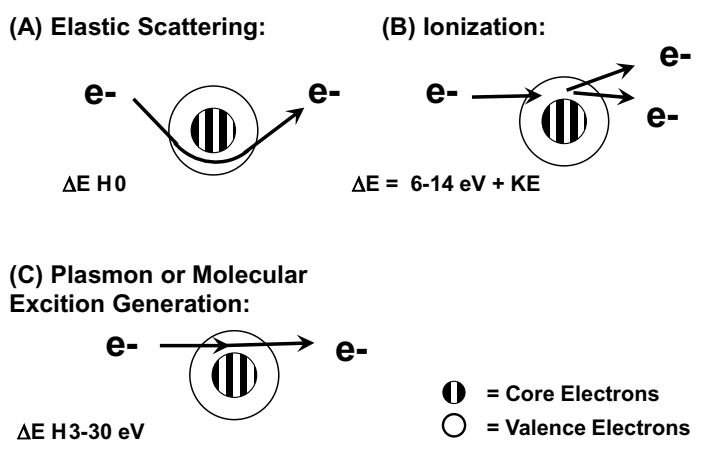

Figure 5. Atomic interactions currently part of the LESiS model: (A) elastic scattering; (B) ionization; and (C) plasmon or molecular exciton generation. A plasmon is a wave of bound valence electrons in a solid. Our model currently tracks only plasmons and not molecular excitons (internal excitation events). a higher-energy vibrational mode. As the molecule returns to its vibrational ground state, this additional energy is released as heat.

\subsection{Monte Carlo Simulation with LESiS}

The code for this Monte Carlo program utilizes an inversion method to determine the probability of elastic and inelastic scattering events occurring using differential cross sections calculated during electron travel with a typical step size of $1 \AA$. The probability of an interaction (or event) for a total travel distance, $S$, and step size, $\Delta \mathrm{x}$, is given by the expressions shown in Figure 6. This method therefore avoids problems with interface artifacts found in mean-free-path-based Monte Carlo codes where a mean free path can be larger than the thickness of thin films typically used in lithography for high energy electrons. The code uses experimental stopping powers as a benchmark, from which the plasmon and ionization contributions are subtracted so that these inelastic processes are simulated stochastically.

The program currently tracks elastic, ionization, and plasmon generation events. The goal for future work is to totally remove the experimental stopping powers mentioned above once HOMO/LUMO transitions (internal excitations) $[23,24], \pi$ plasmons, and vibrational loss differential cross sections can be calculated as a function of incident electron energy.

$$
\begin{array}{ll}
P_{\text {event }}=P_{\text {elastic }}+P_{\text {inelastic }}+P_{\text {plasmon }} & \sigma_{i}=\text { inelastic cross section } \\
P_{\text {inelastic }}=\sigma_{i} e^{-\sigma_{i} s} \Delta x & \sigma_{p}=\text { plasmon cross section } \\
P_{\text {plasmon }}=\sigma_{p} e^{-\sigma_{p} s} \Delta x & \sigma_{e}=\text { elastic cross section } \\
P_{\text {elastic }}=\sigma_{e} e^{-\sigma_{e} s} \Delta x & S=\text { total travel distance } \\
& \Delta x=\text { travel step }
\end{array}
$$

Figure 6 Governing equations. (A) Guiding equations for the inversion method; (B) Mott elastic scattering cross section; (C) modified Grysinski inelastic scattering cross section; and (D) plasmon cross section.

\section{Results and Discussion}

4.1 Simulation Output 
As described above, LESiS follows all elastic and inelastic scattering events between free electrons and resist molecules over a travel step length of $1 \AA$. Since an EUV photon generates a photoelectron in the range of 78-86 $\mathrm{eV}$, we have started our modeling with an $80 \mathrm{eV}$ electron entering a commercial EUV resist [40] perpendicular to the surface. LESiS is capable of mapping the pathway of 10,000 initial $80 \mathrm{eV}$ electrons in approximately 5-10 minutes on a conventional consumer laptop.

Other than the results shown in Figure 8, all modeling experiments were done using the empirical formula of an EUV resist from a commercial supplier. Figure 7A shows the output of a representative LESiS simulation as a three-dimensional electron trajectory map. The incident $80 \mathrm{eV}$ electron generated a single secondary electron which, when both electrons' paths are considered, covered a volume approximately $2 \mathrm{~nm}$ in diameter and $2.5 \mathrm{~nm}$ in height. Most surprising to us from this simulation was that only one daughter electron was generated. After 10,000 runs with these initial conditions, LESiS predicted that the average number of secondary electrons generated per incident electron was $1.8 \pm 1.0$. This result indicates that, on average, there are fewer than two total electrons (and therefore holes) available to initiate the cascade of chemical events leading to acid generation per incident EUV photon. Since the observed quantum yields for iodonium PAGs (4.9 to 5.6 $\mathrm{H}^{+} /$absorbed photon, Figure 2) exceed the predicted number of electron/hole pairs, the model suggests there must be an alternate pathway by which acid can be generated apart from Mechanisms 1 and 2 described above.

\subsection{Secondary electron output dependence on molecular binding energy}

We used the $2 s$ and $2 p$ electron binding energies of all of the elements in the commercial resist to create the electron map shown in Figure $7 \mathrm{~A}$ and to determine the total number of electrons available for reaction with PAG.
However, we recognize that the atomic orbitals with the greatest probability of being ionized are the $2 p$ orbitals of carbon and oxygen, as these are the most abundant second-row elements present in the resist. For example, Figure 7B shows a schematic of the ionization event starting with $2 p$ electrons from carbon and oxygen. The ionization energies for these two atomic orbitals are 9.0 and $14.2 \mathrm{eV}$, respectively; the oxygen $2 p$ orbital requires more energy to ionize than that of carbon because oxygen is more electronegative.

(A)

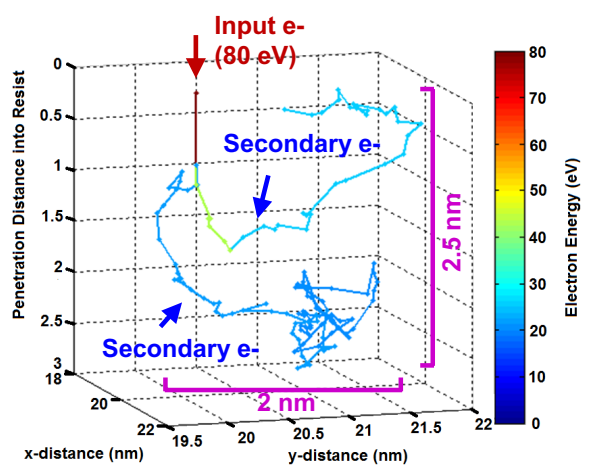

(B)

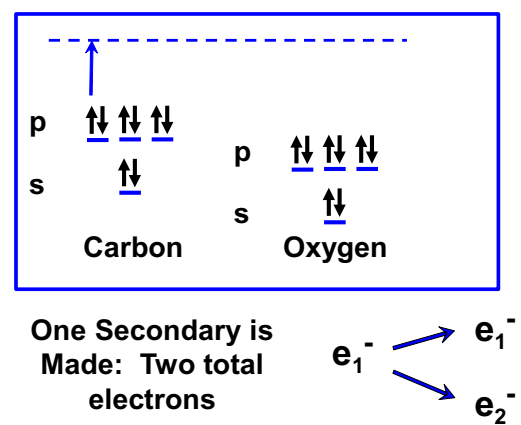

Figure 7. (A) Electron trajectory simulation in a commercial resist. Only ionization event occurs, so there are only two total electrons available to react with PAG. (B) Band gap schematic for carbon and oxygen. Although the model includes the probability of an electron interaction with all of the atomic orbitals in the resist, the highest-energy $2 p$ orbitals have the greatest probability of producing an energy loss event.

To probe the sensitivity of our model to ionization energy, we conducted a study in which we set the binding energies of the $2 p$ electrons of the second-row elements (and the $3 p$ 
orbitals for third-row elements) present in the commercial resist to a single value (e.g. $5 \mathrm{eV}$ ). We ran 500 simulations (incident electron energy $=80 \mathrm{eV}$ ) for each of several binding energies from 1-13 eV and determined the average number of secondary and tertiary electrons generated (Figure 8). As expected, there is a clear inverse relationship between the binding energy and number of electrons produced; that is, more secondary electrons were generated for lower binding energies. For reference, the ionization energies of the carbon and oxygen $2 p$ orbitals and the experimentallydetermined molecular ionization energies for phenol, benzene, and ethyl acetate are also shown in the figure $[38,39]$.

This modeling study predicts that the total number of electrons generated from an absorbed EUV photon would be 2 to 2.3 for the realistic range of ionization energies $(8.5-10 \mathrm{eV})$, which is in general agreement with the electron map approach starting with actual atomic ionization energies of the commercial resist $(1.8 \pm 1.0$ total electrons).

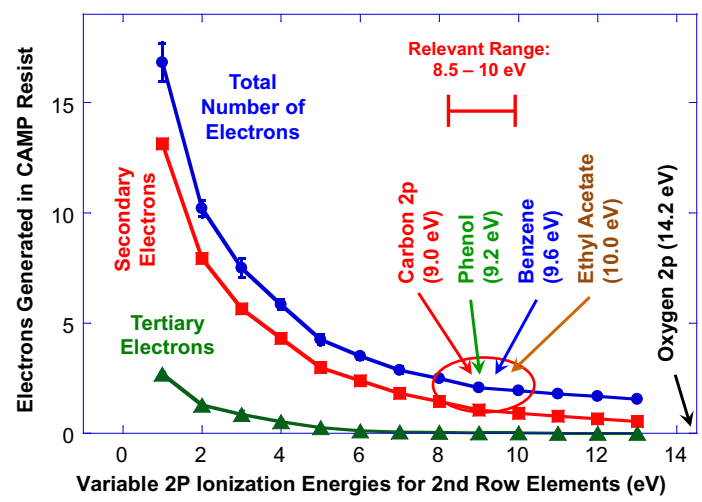

Figure 8. Secondary electron generation dependence on ionization energy. The current LESiS Model predicts that the total number of electrons for our commercial chemically amplified resists will only be 2-2.3 electrons/absorbed photon. Although often smaller than the points, all error bars are shown for the total number of electrons.

\subsection{Mechanisms and FQY Overview}

A comparison between the modeling results above with the results obtained by Higgins $[3,4,6]$ and Kozawa $[8,30]$ in their FQY studies reveals a clear discrepancy if acid generation only occurs through Mechanisms 1 and 2. For both, only ionization events initiate the formation of acid, necessitating a one-to-one correspondence between the number of secondary electrons produced through inelastic scattering and the number of acid molecules generated. If the inherent inefficiency of the chemical reactions is also considered, significantly more than 5-6 electrons would be needed to form the 5-6 acids detected in the FQY studies. However, if our modeling results are correct and only $\sim 2-3$ electrons are produced per photon, there must be an alternative mechanism by which acids are generated without the annihilation of electrons-we propose that these acids are produced through internal excitation (Mechanism 3, Figure 4).

\section{Conclusions and Future Directions}

Though still preliminary, our results demonstrate that previously proposed models of photoacid generation are inadequate to explain the FQY (4.9-5.6 $\mathrm{H}^{+}$/photon) achieved in highPAG resists. Most mechanistic work in this area has focused on the possibility that multiple ionization events are followed by electron recombination with PAG molecules leading either directly (Mechanism 1) or indirectly (Mechanism 2) to acid generation. However, our model predicts that only $\sim 2-3$ electrons are generated per EUV photon, suggesting that there is an alternative mechanism, such as internal excitation, that contributes to acid production.

We have used the Monte Carlo simulation program, LESiS, to analyze the energy loss mechanisms of secondary electrons in EUV resist films. We are in the process of developing the means to add internal excitation events to LESiS v7.71 and we will begin performing modeling studies which begin with EUV photons instead of electrons. Additionally, we hope to equip the program with a graphical user interface to make it more accessible for the resist community. We will also be experimentally examining electron penetration and propagation 
in EUV resists with a variety of spectroscopic techniques, including depth-of-penetration studies using ellipsometry and mass spectroscopy, EELS, and photoelectron spectroscopy. Ultimately we will use these results to validate our model and give us a more comprehensive understanding of acid generation in EUV resist films.

\section{Acknowledgements}

We thank the College of Nanoscale Science and Engineering (CNSE) and specifically, Senior Vice President Alain Kaleyeros for financial support of this project. We also thank the supplier of our commercial resist. Additionally, we thank Brian Cardineau, Bill Earley, Yudhishthir Kandel, Tim Groves, and Alain Diebold for helpful discussions. The use of the Center for Nanoscale Materials, Argonne National Laboratory was supported by the U.S. Department of Energy, Office of Science, Office of Basic Energy Sciences, under Contract No. DE-AC02-06CH11357.

\section{References}

[1] R. Brainard, G. Barclay, E. Anderson and L. Ocola: Microelectronic Engineering. 61-62 (2002) pp 707-715.

[2] Y. Wei and R. Brainard: Advanced Processes for 193-nm Immersion Lithography, SPIE Press, Bellingham, WA, 2009.

[3] R. Brainard, E. Hassanein, J. Li, P. Pathak, T. Wang, B. Thiel, F. Cerrina, R. Moore, M. Rodriguez, B. Yakshinskiy, E. Loginove, T. Madey, R. Matyi, M. Malloy, A. Khurshid, A. Rudack, P. Naulleau, A. Wuest and K. Dean: "Photons, Electrons, and Acid Yields in EUV Photoresists: A Progress Report," Proc. SPIE 6921 (2008).

[4] E. Hassanein, C. Higgins, P. Naulleau, R. Matyi, G. Gallatin, C. Anderson, D. Niakoula, G.Denbeaux, A. Antohe, J. Thackeray, K. Spear, C. Szmanda, M. Malloy, A. Khurshid, C. Montgomery, E. Piscani, A. Rudack, J. Byers, A. Ma, K. Dean and R.Brainard: "Film Quantum
Yields of EUV \& Ultra-High PAG Photoresists," Proc. SPIE 6921 (2008).

[5] J. Biafore, M. Smith, E. van Setten, T. Wallow, P. Naulleau, D. Blankenship, S. Robertson and Y. Deng: "Resist Pattern Prediction at EUV." Proc. SPIE. $\mathbf{7 6 3 6}$ (2010).

[6] C. Higgins, C. Szmanda, A. Antohe, G. Denbeaux, J. Georger and R. Brainard: Jpn. J. App. Phys. 50 (2011) 036504.

[7] T. Kozawa, Y. Yoshida, M. Uesaka and S. Tagawa: Jpn. J. App. Phys. 31 (1992) pp. 4301-4306.

[8] T. Kozawa and S. Tagawa: Jpn. J. App. Phys. 49 (2010) 030001.

[9] A. Nakano, T. Kozawa, K. Okamoto, S. Tagawa, T. Kai and T. Shimokawa: Jpn. J. App. Phys. 45 (2006) 6866-6871.

[10] K. Okamoto, T. Kozawa, K. Natsuda, S. Seki and S. Tagawa: J. Phys. Chem. B. 112 (2008) 9275-9280.

[11] N. Turner and R. Colton: Analytical Chemistry, 54 (1982) 293R-322R.

[12] A. Jablonski: J. Phys. D: Appl. Phys. 45 (2012) 315302 (14 pp).

[13] R. Shimizu and D. Ze-Jun: Rep. Prog. Phys. 55 (1992) 487-531.

[14] R. Shimizu, Y. Kataoka, T. Ikuta, T. Koshikawa and H. Hashimoto: J. Phys. D: Appl. Phys. 9 (1976) 101-114.

[15] J. Ashley, C. Tung, and R. Ritchie: IEEE Transactions on Nuclear Science. NS25 (1978) 1566-1570.

[16] C. Tung, J. Ashley, and R. Ritchie: IEEE Transactions on Nuclear Science. NS26 (1979) 4874-4878.

[17] V. Ivin, M. Silakov, G. Babushkin, B. Lu, P. Mangat, K. Nordquist, and D. Resnick: Microelectronic Engineering. 69 (2003) 594-605.

[18] K. Murata, D. Kyser and C. Ting: J. App. Phys. 52 (1981) 4396-4405.

[19] D. Kyser: J. Vac. Sci. Technol. B. 1 (1983) 1391-1397.

[20] D. Kyser: In Electron Beam Interactions with Solids, ed. by D. Kyser et al. SEM, Inc., AMF O'Hare, Chicago IL. (1982) 331-342.

[21] A. Akkerman and E. Akkerman: J. Appl. Phys. 86 (1999) 5809-5816. 
[22] H. Demers, N. Roirier-Demers, A. Couture, D. Joly, M. Gullmain, N. de Jonge and D. Drouln: Scanning. 33 (2011) 135-146.

[23] L. E. Ocola, F. Cerrina: J. Vac. Sci. Technol. B11 (1993) 2839.

[24] L. E. Ocola: "Electron-matter interactions in $\mathrm{x}$-ray and electron beam lithography". University of WisconsinMadison, 1996.

[25] G. Han and F. Cerrina: J. Vac. Sci. Techno. B. 18 (2002) 3297-3302.

[26] G. Han, M. Khan, Y. Fang and F. Cerrina: J. Vac. Sci. Techno. B. 20 (2002) 2666-2671.

[27] C. Mack, J. Thackeray, J. Biafore and M. Smith: J. Micro/Nanolith. MEMS MOEMS. 10 (2011) 033019.

[28] M. Liehr, P. Thiry, J. Pireaux and R. Caudano: Phys. Rev. B. 33 (1986) 56825697.

[29] M. Vilar and A. Botelho do Rego: Journal of Electron Spectroscopy and Related Phenomena. 172 (2009) 27-35.

[30] R. Hirose, T. Kozawa, S. Tagawa, T. Kai, and T. Shimokawa: Appl.Phys. Express. 1 (2008) 027004.

[31] J. Thackeray, J. Cameron, V. Jain, P. LaBeaume, S. Coley, O. Ongayi, M. Wagner, A. Rachford and J. Biafore: "Progress in
Resolution, Sensitivity and Critical Dimensional Uniformity of EUV Chemically Amplified Resists." Proc. SPIE 8682 (2013).

[32] T. Kozawa, T. Shigaki, K. Okamoto, A. Saeki, S. Tagawa, T. Kai, and T. Shimokawa: J. Vac. Sci. Technol. B 24 (2006) 3055.

[33] J. Ritsko, L. Brillson, R. Bigelow and T. Fabish: J. Chem. Phys. 69 (1978) 3931-3939.

[34] J. Ritsko: J. Chem. Phys. 70 (1979) 5343-5349.

[35] R. F. Egerton: Rep. Prog. Phys. 72 (2009).

[36] D. Tahir and S. Tougaard: J. Appl. Phys. 111 (2012) 054101.

[37] B. Reznik and M. Fotouhi: Composites Science and Technology. 68 (2008) 11311135.

[38] G. Crable and

G. Kearns: J. Phys.Chem. 66 (1962) 436439.

[39] D. Lide, ed. Handbook of Chemistry and Physics. $83^{\text {rd }}$ edition. CRC Press, New York, 2002, p. 10-191

[40] We used the confidential empirical formula supplied by the resist vendor and used a mass density of 1.4 based on previous work (Brainard and Higgins). 